\title{
An Appraisal of Antibiotic Policies for Urinary Tract Infections in Patients with Spinal Cord Injuries Undergoing Long-term Intermittent Catheterisation
}

\author{
D. J. Stickler, BSc, MA, DPhil ${ }^{1}$, J. C. Chawla, MD, FRCS ${ }^{2}$ \\ ${ }^{1}$ Department of Applied Biology, UWIST, Cardiff, CF1 3XF, UK, ${ }^{2}$ Spinal \\ Injuries Unit, Department of Rehabilitation Medicine, Rookwood Hospital, \\ Cardiff, CF5 2 YN, UK.
}

\begin{abstract}
Summary
The antibiotic policies that have been recommended in the literature for the prevention and treatment of urinary tract infections in patients with spinal cord injuries undergoing long-term intermittent catheterisation are reviewed. Current practices in spinal units in England and Wales are reported and the rational bases of these policies are considered.
\end{abstract}

Key words: Spinal cord injured patients; Urinary tract infections; Intermittent catheterisation; Antibiotic policies.

The preservation of renal function is an important objective in the care of patients with a neuropathic bladder due to a spinal cord injury. Although there has been substantial improvement in the urological management, urinary tract infection and deterioration in renal function is still a major cause of morbidity and mortality in these patients (Grundy and Russell, 1986).

The early management of patients in a spinal injury unit generally involves the use of intermittent urethral catheterisation. The technique is performed by staff and is repeated 4-6 hourly. It is also appropriate for the long-term management of those patients who retain adequate hand function and can perform the technique themselves. Many authorities have written about their clinical experience with intermittent catheterisation and expressed their views that its use had lowered rates of urinary infection, reduced the incidence and severity of urological complications, and allowed a higher percentage of patients to achieve a catheter-free state (Guttmann and Frankel, 1966; Wilmot and O'Flynn, 1967; Ott and Rossier, 1971; Erickson et al., 1982; Joiner and Lindan, 1982; Pearman, 1984). Warren et al., (1981) in reviewing the relative merits of intermittent and indwelling catheterisation pointed out that it is difficult to determine the incidence of infection from many papers on intermittent catheterisation and that such an analysis is confused by the routine use of prophylactic systemic antibiotics in many spinal units. It is quite clear however, that most patients on long-term intermittent catheterisation develop urinary infections and many suffer recurrently or sometimes continuously infected 
urines (Vainbrub and Musher, 1977; Donovan et al., 1978; Moloney et al., 1981; Erickson et al., 1982; Krebs et al., 1984; Cardenas and Mayo, 1987). The purpose of this article is to review the antibiotic policies that have been recommended in the literature for the prevention and treatment of these urinary tract infections; to report on current practices in spinal units in England and Wales and to consider the rational bases of these policies.

\section{Prophylaxis}

In their recent book The Urinary Tract and the Catheter Slade and Gillespie (1985) commented that because of the special danger of renal infection, longterm prophylactic chemotherapy should be used with patients on intermittent catheterisation. The agents that have been recommended for this purpose include methenamine, ascorbic acid, nitrofurantoin, trimethoprim and cotrimethoxazole.

Methenamine is given orally as methenamine mandelate or hippurate and Slade and Gillespie (1985) emphasise that it should be administered under carefully controlled conditions. Fluid intake should not be excessive and daily urine volume should be 2 to 2.5 litres. The $\mathrm{pH}$ of the urine should be checked daily and if it rises above $\mathrm{pH} 6.0$ then an acidifying agent such as ammonium chloride or methionine, should be added to the diet. Alternatively the urine may be kept acid by a high protein diet that is low in milk products. Pearman and England (1976) recommended that if methenamine did not prevent bacteriuria or produced gastro-intestinal upsets, then nitro-furantoin (50-100 mg) once daily at bedtimes should be tried. Joiner and Lindan (1982) reported that in their unit it is the usual practice to use low doses of antibacterial drugs during intermittent catheterisation, methenamine, nitrofurantoin or co-trimethoxazole being the agents employed. At the Mayo Clinic most patients on intermittent catheterisation are given the systemic bacterial suppressants methenamine or cotrimethoxazole (Viera et al., 1986). Grundy and Russell (1986) suggested that for those patients who suffer recurrent urinary tract infections, acidification of the urine using a non-effervescent ascorbic acid preparation, or the use of methenamine may be beneficial. They also added that a high urinary output of at least 3 litres in 24 hours also helps to minimise infection.

Our enquiries have revealed that five distinct prophylactic policies are operated in the spinal units of England and Wales:

1. No prophylactic agents used for any patients.

2. All patients receive daily ascorbic acid to acidify the urine.

3. Only patients with recurrent significant bacteriuria and pyuria or symptomatic infections are given ascorbic acid or methenamine.

4. Patients with recurrent significant bacteriuria and pyuria or symptomatic infections are given long term cephradine/trimethoprim/septrin or ciprofloxacin.

5. Patients with recurrent symptomatic infection have long-term ascorbic acid and methenamine or trimethoprim or nitrofurantoin. 


\section{The rational bases of the prophylactic policies}

Ascorbic acid. It is still common to prescribe daily doses of vitamin C (2-4 grammes) for patients with spinal injuries as a urinary acidifying agent. Several investigations have however demonstrated, that ascorbic acid does not significantly lower the urinary $\mathrm{pH}$. In normal male volunteers McLeod and Nahata

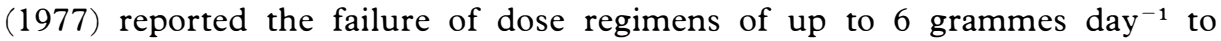
significantly change urinary $\mathrm{pH}$. Hetey et al. (1980) conducted a detailed study in which ascorbic acid ( 1 gramme four times daily) or placebo was administered for 5 days in a double-blind, cross-over study to 20 patients with spinal cord injury and neurogenic bladder. The urinary $\mathrm{pH}$ was measured 4 times daily for 2 days before and then during the administration of the ascorbic acid or placebo. The mean urinary $\mathrm{pH}$ before the treatment ranged from 5.43 to 6.51. During the study the mean $\mathrm{pH}$ of urine while the patients were being given ascorbic acid ranged from 5.06 to 6.82 and from 5.25 to 6.85 while being given placebo. Analysis of variance on the data revealed no significant differences in these three sets of means. Lamid (1983) reported a similar study in which 6 patients with spinal cord injuries were given $500 \mathrm{mg}$ of ascorbic acid 4 times daily. Before the medication the mean urinary $\mathrm{pH}$ was 6.83 , 1 hour after medication the mean $\mathrm{pH}$ was 6.23 and 2 hours later 7.00. Statistical analysis revealed no significant differences between these mean values.

Devenport et al., (1984) found that ascorbic acid (1 gramme 4 times daily) changed the urinary $\mathrm{pH}$ in spinal patients undergoing intermittent or indwelling catheterisation from mean values of $6.16 \pm 0.75$ to only $5.82 \pm 0.94$ and concluded that this agent could not be recommended as a urinary acidifier. Kuhlemier et al., (1986) compared the incidence of urinary tract infection in patients given no prophylactic agents to those receiving ascorbic acid and reported that those receiving ascorbic acid generally had shorter infection free periods than the control group.

Methenamine. The bactericidal action of methenamine results from its hydrolysis in acid urine to formaldehyde. Theoretically it has many of the properties which make it a highly suitable agent for long-term prophylaxis. It has a broad spectrum of action and bacterical resistance to it does not develop. It only becomes active after it has been excreted into the urine and so has no adverse effects on the normal flora of the gut. Another important factor is that it is a relatively inexpensive agent. While it is commonly used as a bacterial suppressant for chronically catheterised patients there is little evidence that it is effective. Vainrub and Musher (1977) examined the ability of methenamine mandelate and ascorbic acid ( 1 gramme of each 4 times daily) to prevent urinary infection in 9 patients undergoing intermittent catheterisation. All patients became infected, the mean interval between the initial uninfected specimen and infection being 11.7 days. As we have seen however, ascorbic acid is a poor choice as a urinary acidifier. Devenport et al. (1984) in their study which demonstrated only a small change in urinary $\mathrm{pH}$ on administration of ascorbic acid also showed that when these patients were also given methenamine mandelate or hippurate the ascorbic acid did not significantly change the concentration of formaldehyde in the urine. The mandelate was shown to produce mean urinary concentrations of formaldehyde of $47 \mu \mathrm{gml}^{-1}$ while the hippurate 
generated a mean concentration of $25 \mu \mathrm{gml}^{-1}$ (a highly significant difference $\mathrm{p}<0.001)$. Devenport et al. (1984) concluded that mandelate was preferable to hippurate for the suppression of bacteriuria in spinal injured patients but that ascorbic acid could not be recommended as a urinary acidifier.

A more effective urinary acidifier seems to be ammonium chloride. Pearman et al., (1978) reported a study in which catheter-free paraplegic patients given methenamine mandelate $(4 \times 1$ gramme daily) produced urine with a mean $\mathrm{pH}$ of 6.6 When the methenamine was supplemented with ammonium chloride $(4 \times$ 1 gramme daily) the mean urinary $\mathrm{pH}$ dropped to 5.0 and the mean concentration of urinary formaldehyde rose from 8 to $41 \mu \mathrm{gml}^{-1}$. The antibacterial activity of the urines was examined against Escherichia coli, Pseudomonas aeruginosa, Streptococcus faecalis and Proteus mirabilis. Urine from patients receiving methenamine and ammonium chloride generally sterilised inocula of $c a 10^{5}$ bacteria $\mathrm{ml}^{-1}$ within 6 hours while no antibacterial activity was detected in urine of patients just receiving methenamine. Unfortunately the antibacterial activity of urine from patients given ammonium chloride alone was not tested, so the effect of urinary acidity was not isolated from the combined effects of methenamine and acidity. An examination of their data however, reveals that the greatest bactericidal activity was exhibited by the most acidic urine which had the lowest formaldehyde concentration. In this study the ammonium chloride was only administered for 3 day periods and the authors suggested that because of the possibility of physiological adaptation in the kidney to neutralise long-term urinary acidity, any programme in which this medication is used should involve the regular monitoring of urinary $\mathrm{pH}$.

An alternative approach to urine acidification has been taken by Krebs et al., (1984). In view of the poor antibacterial activity of urine from patients receiving methenamine mandelate and ascorbic acid, they investigated the effectiveness of a regime in which oral methenamine was supplemented by bladder instillations of $5^{\circ}{ }_{0}$ hemiacidrin, a solution of citric and gluconic acids at pH 3.9 (Mulvaney, 1959), at each catheterisation. Forty men, admitted to a spinal cord injury service were randomly assigned to 2 prospective study groups. The control group underwent intermittent catheterisation without any antibacterial prophylaxis, while the treated group received methenamine $(4 \times 1$ gramme daily) and hemiacidrin bladder instillations $(45 \mathrm{ml})$. The mean $\mathrm{pH}$ of urines produced by the control group $(5.9)$ was significantly higher $(\mathrm{p}<0.01)$ than that recorded in the treated group (5.3). Over mean study periods of 71 days for the controls and 74 days for the treated group, the incidence of significant bacteriuria $\left(>10^{5}\right.$ bacteria $\mathrm{ml}^{-1}$ ) was $70^{\circ}$ o in the control group and $34^{\circ}{ }_{0}^{\circ}$ in the treated group. In addition the number of symptomatic infections was reduced from 21 in the control group to 4 in the treated group. Despite their observation that $25^{\circ}{ }_{0}$ of the treated patients experienced gross haematuria they concluded that the combination of oral mandelamine and bladder instillation of hemiacidrin was a safe and effective means of reducing pyelonephritis in spinal cord patients on intermittent catheterisation.

Again it is clear that Krebs et al., (1984) were observing the combined effect of mandelamine and urinary acidification and did not examine the effect of urinary acidification in isolation. They recalled however that earlier workers (Fam et al., 1976) had noted that acidification of urine by hemiacidrin in order 
to dissolve calculi also seemed to have a suppressive effect on bacterial growth. There is clearly room for a study of the prophylactic effect or urinary acidification in these patients.

\section{Antibiotics}

A review of the literature on the use of prophylactic antibiotics reveals that none of the studies have demonstrated a clear beneficial effect on the incidence of urinary infection. Anderson (1980a) examined the effect of prophylaxis on the rates of bacteriuria in patients on intermittent catheterisation performed by genitourinary technicians using sterile techniques in the acute stages of neurogenic bladder. Patients (64) were randomly assigned to 1 of 4 groups. Group 1 received no prophylactics; group 2 received $30 \mathrm{ml}$ of neomycin/polymyxin as a bladder instillation (Neosporin GU Irrigant) at each catheterisation, group 3 had single daily oral doses of nitrofurantoin $(100 \mathrm{mg}$ ) and group 4 had nitrofurantoin plus the bladder instillation. Bacteriuria was monitored with daily dip-strip cultures and patients were designated as infected if two consecutive cultures had colony counts $>10^{4} \mathrm{ml}^{-1}$. Over the study period 2568 daily cultures were taken and only 83 incidents of significant infection were recorded, 36 of these were in the control group, 20 in the bladder instillation group, 18 in those receiving nitrofurantoin and only 9 in those having both oral nitrofurantoin and the bladder instillation. The most commonly isolated bacterium however was Staphylococcus epidermidis, an organism not normally thought to pose a threat to the urinary tracts of these patients. If the incidents of infection with Staph. epidermidis are eliminated from the data, the difference in infection rates between the control and the nitrofurantoin group disappears. In addition the difference in rates between the controls and those receiving nitrofurantoin and bladder instillation can be accounted for by the effect of the neomycin/polymyxin.

In a subsequent study Anderson (1980b) reported on his experience with 25 acute male spinal patients on clean intermittent catheterisation plus the prophylactic regimen of oral antibiotic and bladder instillation and observed that rates of infection were not significantly different from a group of 16 control patients on clean intermittent catheterisation and no prophylaxis.

Merritt et al. (1982) reported on the rates of urinary infection $\left(>10^{4}\right.$ bacteria $\mathrm{ml}^{-1}$ urine) in patients receiving (a) methenamine hippurate and ascorbic acid (b) co-trimethoxazole and (c) no prophylactic suppressants. The 16 patients in the study were examined for significant bacteriuria at 3 follow-up investigations during a 3 year period. The number of incidents of infection recorded in the groups were 2, 0 and 6 respectively. The authors expressed the opinion that the long-term use of suppressants can help to reduce the rates of urinary tract infection in patients emptying their bladders by intermittent self-catheterisation. The authors, themselves, however pointed out that the numbers of patients in each group (5 and 6) were too small to allow a statistical check on the relative efficacy of the treatments.

Lindan and Joiner (1984) reported that their experience with co-trimethoxazole as a prophylactic was that a drug resistant 'enterobacter' emerged as a common urinary organism. They therefore investigated nitrofuratoin (50 
mg twice daily) as an alternative. In a prospective study, patients were assigned to a control group in which antibiotics were only given when urinary infection was diagnosed, or to a test group who received the prophylactic nitrofurantoin. The criteria for urinary tract infection were pyuria and bacteriuria $\left(>10^{5}\right.$ organism $\mathrm{ml}^{-1}$ ) with 'ureopathic' organisms. Those organisms considered to be significant were E. coli, Proteus, Klebsiella and Enterobacter. Sixty patients were studied for an average time of 11.8 days, Intermittent catheterisation was used with 27 of the patients, indwelling catheters with 25 and the remaining 8 were not undergoing catheterisation. The results were quoted for the whole group of 60 . In the control group 25 patients experienced a total of 60 episodes of infection; of those receiving prophylactic nitrofurantoin 8 patients suffered a total of 9 infections. The authors do mention however that Pseudomonas was isolated from 23 patients in the treated group. They considered that this organism was a harmless coloniser that could be 'safely left to browse undisturbed in the neuropathic bladder'. This exclusion of Pseudomonas infections from the analysis of the data is a contentious step, as it is generally thought to be an important pathogen in the catheterised urinary tract. The evidence for Lindan and Jones's distinction between 'true pathogens' and 'colonisers' was based on the results of tests for antibody coated bacteria in patients urine. Unfortunately this test has been shown to be an unreliable index of invasive infection of the urinary tract of catheterised patients (Merritt and Keys, 1982).

Maynard and Doikno (1984) in their study on the effect of prophylactic antibiotics for patients using clean intermittent catheterisation, distinguished between 'laboratory' diagnosed infections and 'clinical' infections. The criteria for a 'laboratory' infection were $(a) \geqslant 25$ pus cells/HPF of urine and $>10^{5}$ bacteria $\mathrm{ml}^{-1}$ urine without fever or urethritis (b) $\geqslant 25$ pus cells/HPF and $>10^{4}$ bacterial $\mathrm{ml}^{-1}$ urine on 2 or more consecutive samples (c) bacteriuria $>10^{5}$ organisms $\mathrm{ml}^{-1}$ with the same organism on 2 consecutive bi-weekly urine cultures. The criteria for clinical urinary infection were (a) temperature $>100^{\circ} \mathrm{F}$ and either $>10^{5}$ bacteria $\mathrm{ml}^{-1}$ urine or $>25$ pus cells/HPF (b) purulent uretheral discharge and positive urethral culture (c) other clinical signs indicating abdominal or pelvic irritation (lower abdominal pain, reflex sweating and increased spasticity) occurring when criteria for laboratory infections were met. Fifty patients on clean intermittent catheterisations were studied for a mean period of 50 days. During this time $68^{\circ}{ }_{0}$ of the patients had infections which met the laboratory criteria and $22^{\circ}{ }_{0}$, had clinical urinary tract infections. The daily probability of the development of a 'laboratory' or 'clinical infection for control subjects and those given prophylactic therapy (trimethoprim $80 \mathrm{mg}$, sulpha-methoxazole $400 \mathrm{mg}$ daily or nitrofurantoin $(100 \mathrm{mg})$ if they were allergic to sulphonamides, was determined and analysis of the data showed that antibacterial prophylaxis significantly reduced the probability of 'laboratory' but not that of 'clinical' infections.

Dean (1986) failed to demonstrate any significant difference in the incidence of bacteriuria ( $>10^{4}$ organisms $\mathrm{ml}^{-1}$ urine) in a small group of patients who were randomly assigned to receive no prophylactics or to receive co-trimethoxazole for a month followed by nitrofurantion for a further month. Over the period of the study 18 episodes of significant bacteriuria were recorded in the controls and 17 in the treated patients. 
The long-term preservation of renal function is the main concern in the urological care of these patients and the effect of the various prophylactic regimes on this important criterion does not appear to have been investigated. Viera et al., (1986) however recently reported that $21^{\circ}{ }^{\circ}$, of a group of patients on intermittent catheterisation who were being given prophylactic methenamine or co-trimethoxazole, developed abnormal excretory urograms over a period of 30 months.

\section{Treatment}

A variety of advice is available in the literature on how and when to treat urinary infections in these patients. Anderson (1980) proposed that bacteriuria ( $>10^{4}$ organisms $\mathrm{ml}^{-1}$ on 2 successive days) should be treated with the appropriate antibiotic for 5 days, and that an infection associated with a high fever should be considered as pyelonephritis and treated more aggressively with parenteral antibiotics for 10-14 days. Perkash and Giroux (1985) have adopted a policy in which antibiotic treatment is given (a) when a patient is suffering from fever, chills, signs of dysreflexia or reflux, (b) when the infecting organism is a urea splitter i.e. Proteus or Providencia and (c) when there is evidence of an inflammatory response in the asymptomatic patient as indicated by $>10^{4}$ w.b.c. $\mathrm{ml}^{-1}$ along with $>10^{5}$ bacteria $\mathrm{ml}^{-1}$ urine. They added that aminoglycosides and other broad spectrum antibiotics are only used if expressly indicated.

The advice of Grundy and Russell (1986) was that infections producing systemic effects or the presence of $>10^{5}$ organisms $\mathrm{ml}^{-1}$ or $>50$ w.b.c. per high power field, should be treated with the appropriate drug. They also made the point that it is particularly important to irradicate Proteus $s p$.

Slade and Gillespie (1985) expressed the opinion that everything possible must be done to prevent bacteriuria in these patients. Urinary infection (criteria not defined) should be treated by appropriate antibiotics selected from the results of recent urine culture. When the infection has been irradicated they suggest that a prophylactic regime of methenamine, trimethoprim or nitrofurantoin should be instituted. Galloway et al. (1986) have recently pointed out however, that pressures to limit the use of antibiotics because of the threat of emergence of resistant organisms, the risk to patients from unwanted side effects of antibiotics, the expense and the risk to other patients from cross-infection with resistant organisms has commonly led to the withholding of antibiotic therapy until clinical symptoms indicate that the upper urinary tract has become involved in the infection.

Our review of current practice in spinal units in England and Wales has confirmed that a variety of policies are employed, the most popular option however, is indeed to withhold therapy until clinical symptoms appear. In some units the presence of significant bacteriuria together with pyuria is the signal to start therapy. Significant bacteriuria $\left(>10^{5}\right.$ organisms $\mathrm{ml}^{-1}$ ) in the absence of pyuria is treated in some units especially if the infecting organisms are Proteus or Pseudomonas. The selection of antibiotics for non-symptomatic infections is generally based on the results of recent sensitivity tests. Symptomatic infections are usually treated with broad-spectrum cephalosporins or aminoglycosides. There is concern in some quarters about the common usage of gentamicin in 
view of its renal toxicity and the increasing incidence of resistant strains. For these reasons one unit practices severe restriction in the use of this drug.

It is difficult to assess the relative merits of the various policies as little or no comparative work on their efficacy has been reported in the literature. The early work of Donovan et al. (1978) was designed to characterise the urinary populations in these patients and observe how they changed in response to chemotherapy. They examined a group of 60 patients who were catheterised using a sterile technique at 4-6 hour intervals. All patients received ascorbic acid and most also were given methenamine mandelate but no antibiotics were used prophylactically. Daily bacteriology was performed on the urines over a mean study period of 13.5 weeks per patient. The definition of bacteriuria was taken as the appearance of the same organism on two successive days with no minimum colony count. A total of 178 episodes of bacteriuria were encountered and the commonly appearing organisms were E. coli $\left(29^{\circ}{ }_{0}\right)$, Klebsiella-Enterobacter $\left(25^{\circ}{ }_{0}\right)$, Pseudomanas $\left(18^{\circ}{ }_{0}\right)$, Enterococcus $\left(17^{\circ}{ }_{0}\right)$, Proteus mirabilis $\left(7^{\circ}{ }_{0}\right)$ and Candida albicans $\left(3_{0}^{\circ}\right)$. These episodes were treated with appropriate antibiotics for 2 week periods. Bacteriuria with the same or different organism commonly reappeared within 5 days from discontinuation of therapy. This finding prompted the authors to suggest a 6 week treatment period in an effort to thoroughly irradicate offending organisms, but this was not investigated further.

Support for such a policy has come from recent anatomical studies on infected human bladders using scanning electron microscopy (Elliot et al., 1986). Electron micrographs of bladder epilithelia from patients who had suffered chronic urinary tract infection showed gross disturbance of the urothelium with extensive exfoliation and exposure of underlying cells. Elliot and colleagues suggested that long-term treatment may be required for the chronically infected bladder in order to allow regeneration of the urothelium, a process which may take up to 6 weeks. Such a policy might be particularly appropriate for intermittently catheterised patients where reinfection of the urinary tract commonly occurs on completion of the normal course of antibiotic therapy.

Maynard and Doikno (1984) reported no apparent benefit from the prompt antibiotic treatment of asymptomatic bacteriuria with and without pyuria in a group of patients on clean intermittent catheterisation. The incidence of clinical symptomatic infections in these patients was not significantly different from that in a group who were not treated. They suggested however that follow-up studies are needed to ensure that policies limiting the use of antibiotics are associated with a low probability of severe urological complication in the longterm.

Galloway et al., (1986) came to the conclusion that to protect the integrity of the urinary tract there are occasions on which it is necessary to treat asymptomatic infections. They pointed out that clinical signs are notoriously unreliable for the diagnosis of urinary tract infections, especially in this group of patients where disturbance of sensation or thermoregulation may mask the classical symptoms of pyelonephritis. In attempt to differentiate between colonisation of the bladder and invasive urinary tract infection, they determined serial serum concentration of C-reactive protein (CRP) an acute phase indicator of inflammation. A group of 16 patients were examined over a period in which 12 episodes of symptomatic 
infection occurred. In each of these cases the serum CRP was elevated and fell back to normal on antibiotic treatment. A particularly interesting observation was that during the study, 6 of the patients also showed elevated serum CRP when they were clinically well and the only known site of infection or inflammation was the urinary tract. They therefore suggested that an increased serum CRP in the presence of asymptomatic bacteriuria and the absence of other known sites of sepsis or infection indicates an invasive urinary tract infection and as such requires treatment with antibiotics. Conversely a normal level of CRP in the presence of bacteriuria indicates a simple bladder colonisation and does not require antibiotics. Serum CRP determinations clearly provide information that would be useful in the management of these patients, however the requirement for regular blood samples and the expense of the assay present difficulties for routine use.

A simple non-invasive test to localise the level of urinary tract infections, that could be performed on urine would be a real asset in the care of these patients. It is unfortunate that the detection of antibody-coated bacteria in the urine has proved to be an unreliable indicator of pyelonephritis in these patients (Merritt and Keys 1982).

The identification of certain organisms such as Proteus, Klebsiella and Pseudomonas as particularly important threats to the integrity of the urinary tract, warranting their erradication from urine in the absence of symptoms stems from their perceived association with alkali urines, the deposition of urinary salts and the development of calculi. While it is an interesting concept that some bacteria are more virulent and invasive than others, few studies have examined as association between clinical symptoms, the deterioration in renal function and any particular bacterial species. In non-catheterised patients, it is now well established that a special group of $E$. coli strains possessing a collection of virulence makers are generally responsible for pyelonephritic infections (Winberg, 1986). It is not known whether these strains occur in the urines of the long-term catheterised patients. If they do then it would be important to establish whether they are particularly associated with kidney infections and as such whether they should be major targets for chemotherapy. The early detection and elimination of such strains could well assist in preserving renal integrity in these patients.

\section{Conclusions}

In 1981 Warren et al., commented that the role of antibiotics in the prevention and treatment of urinary tract infections in patients on long-term intermittent catheterisation had not been adequately defined. Our review of current practices suggests that the situation has not changed substantially and that there is still uncertainty about when and how to use antibacterials in this context. In our experience factors such as prolongation of the interval between catheterisations, traumatic catheterisation or occurrence of vesico-uretheral reflux can preciptiate episodes of symptomatic pyelonephritis or bacteraemia. Under these circumstances whatever organisms happen to be colonising the bladder at the time will be the infecting organism that have to be dealt with by antibiotics. The important factor here is that these bacteria should not be multi-drug resistant and 
should be susceptible to chemotherapy. Limitation in the use of antibiotics is clearly necessary to achieve this situation and therefore we suggest that it important to avoid the prophylactic use of antibiotics for patients with longterm intermittent catheterisation.

There are other instances when clinical symptoms suggesting pyelonephritis or bacteraemia occur in the absence of any obvious precipitating factor and these episodes are presumably brought about by the innate virulence of certain urinary organisms. The development of simple, rapid tests to identify these virulent strains might enable their early detection and elimination by chemotherapy, prior to their invasion of urinary tract tissue.

For those patients who suffer recurrent symptomatic infections it would be well worthwhile investigating the value of 6 week periods of chemotherapy to allow time for the regeneration of damaged tissue before exposing the tract to bacterial recolonisation.

Whether to treat patients who have asymptomatic bacteriuria and pyuria is a contentious issue. The balance of opinion in England and Wales is that treatment under these circumstances is of no value. Because of the need to limit the use of antibiotics we do not recommend treatment in these cases. We feel however that there is a need for further investigation to identify those organisms which have a high probability of producing subsequent symptomatic infections and complications. We also agree with Galloway et al., (1986) that there is a need to develop a simple laboratory test to locate the site of infection in order to provide assurance that asymptomatic pyelonephritis is not undermining renal integrity in these patients.

\section{References}

ANDERSON RU 1980a. Prophylaxis of bacteriuria during intermittent catheterisation of the acute neurogenic bladder. Fournal of Urology 123:364-366.

ANDERSON RU 1980b. Non-sterile intermittent catheterization with antibiotic prophylaxis in the acute spinal cord injured male patient. Fournal of Urology 124:392-394.

CARDENAS DD, Mayo ME 1987 Bacteriuria with fever after spinal cord injury. Archives of Physical Medicine and Rehabilitation 68:291-293.

DEAN LS 1986 Suppressive antibacterial therapy in the prevention of urinary tract infection in spinal cord injury patients. Paraplegia 24:60.

Devenport JK, Senson JR, Dukes GE, Sonsalla PK 1984. Archives of Physical Medicine and Rehabilitation 65:257-259.

Donovan WH, Stolov WC, Clowers DE, Clowers MR 1978 Bacteriuria during intermittent catheterisation following spinal cord injury. Archives of Physical Medicine and Rehabilitation 59:351-357.

Elliot TSJ, SlaCk RCB, BISHOP MC 1986 Scanning electron microscopy and bacteriology of the human bladder in acute and chronic urinary tract infections. In: AssCHER AW, BRUMFITT W (eds), Microbial Diseases in Nephrology, J Wiley and Sons, Chichester, pp. 31-46.

ERICKSON RP, MERritt JL, OPtiz JL, ILSTRUP DM 1982 Bacteriuria during follow-up in patients with spinal cord injury. Archives of Physical Medicine and Rehabilitation 63:409-412.

FAM LB, Rossier AB, Yalla S, Berg G 1976 The role of hemiacidrin in the management of renal stones in spinal cord injury patients. Fournal of Urology 116:696-698.

Galloway A, Green HT, Windsor JJ, Menon KK, Gardner BD 1986 Serial concentrations of C-reactive protein as an indicator of urinary tract infection in patients with spinal injury. fournal of Clinical Pathology 39:851-855.

GRUNDY D, RusSELl J 1986 ABC of spinal cord injury: urological management. British Medical fournal 292:249-253.

Guttmann L, Frankel H 1966 The value of intermittent catheterisation in the early management of traumatic paraplegia and tetraplegia. Paraplegia 4:63-84. 
Hetey SK, Kleinberg ML, Parker WD, Johnson EW 1980 Effect of ascorbic acid on urine pH in patients with injured spinal cords. American fournal of Hospital Pharmacy 37:235-237.

JOINER E, LINDAN R 1982 Experience with self-intermittent catheterisation for women with neurological dysfunctions of the bladder. Paraplegia 20:147-153.

Krebs M, Halvorsen RB, Fishman IJ, Santoz-Mendoza N 1984 Prevention of urinary tract infection during intermittent catheterisation. Fournal of Urology 31:82-85.

Kuhlemeier KV, Stover SL, Lloyd LK 1986 Prophylactic antimicrobial therapy for preventing urinary tract infection after spinal cord injury. Paraplegia 24:59.

LAMID 1983 Ascorbic acid and methenamine mandelate on the urinary $\mathrm{pH}$ of spinal cord injury patients. Fournal of Urology 129:845-846.

LINDAN R, JOINER E 1984 A prospective study on the efficacy of low dose nitrofurantoin in preventing urinary tract infections in spinal cord injury patients with comments on the role of pseudomonads. Paraplegia 22:61-65.

MAYNARD FM, DoIknO AC 1984 Urinary infection and complicatons during clean intermittent catheterisation following spinal cord injury. Fournal of Urology 132:943-946.

MCLeod DC, Nahata MC 1977 Inefficacy of ascorbic acid as a urinary acidifier. New England Fournal of Medicine 296:143.

Merritt JL, ERICKSON RP, Optiz JL 1982 Bacteriuria during follow-up in patients with spinal cord injury. Archives of Physical Medicine and Rehabilitation 63:413-415.

MERRITT JL, KEYS TF 1982 Limitations of the antibody coated bacteria test in patients with neurogenic bladders. Fournal of the American Medical Association 247:1723-1725.

Moloney PJ, Doyle AA, Robinson BL, Fenster H, McLoughlin HG 1981 Pathogenesis of urinary infection in patients with acute spinal cord injury on intermittent catheterisation. Fournal of Urology 125:672-673.

MULVANEY WP 1959 A new solvent for certain urinary calculi: a preliminary report. fournal of Urology 82:546-548.

Otт R, Rossier AB 1971 The importance of intermittent catheterisation in bladder re-education of acute traumatic spinal cord lesions In: Proceedings of the Eighteenth Veterans Administration Spinal Injury Conference pp 139-148.

PEARMAN JW 1984 Infection hazards in patients with neuropathic bladder dysfunction. fournal of Hospital Infection 5:355-358.

Pearman JW, Peterson GJ, Nash JB 1978 The antimicrobial activity of urine of paraplegic patients receiving methenamine mandelate. Investigative Urology 16:91-98.

PERKASH I, GiROUX J 1985 Prevention, treatment and management of urinary tract infections in neuropathic bladders. Fournal of the American Paraplegic Society 8:15-17.

Slade N, Gillespie WA 1985. The urinary Tract and the Catheter J. Wiley and Sons, New York.

VAINBub B, Musher DM 1977 Lack of effect of methenamine in supression of or prophylaxis against chronic urinary infection Antimicrobial Agents and Chemotherapy 12:625-629.

Viera A, Merritt JL, Erickson RP 1986 Renal function in spinal injury. Archives of Physical medicine and Rehabilitation 67:257-259.

WARren JW, Muncie HL, Bergquist EJ, Hoopes JM 1981 Sequelae and management of urinary infection in the patient requiring chronic catheterisation. Fournal of Urology 125:1-8.

Wilmot CB, O'FLYNN JC 1967 Continuous or intermittent catheter drainage in early paraplegia. Urology Digest 6:17-20.

WINBERG J 1986 Balance between host defence and bacterial virulence in urinary tract infection. In: Asscher AW, Brumfitt W (eds), Microbial Diseases in Nephrology, J Wiley and Sons, Chichester, pp 197-224. 